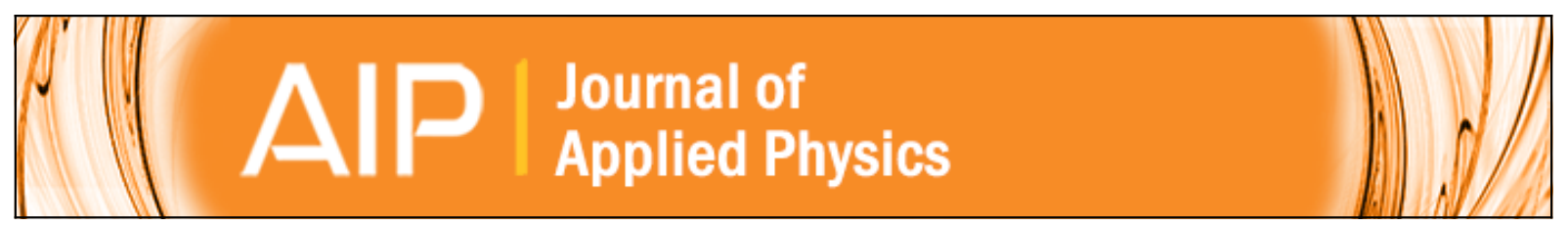

\title{
Thermal transport through short-period SiGe nanodot superlattices
}

Peixuan Chen, J. J. Zhang, J. P. Feser, F. Pezzoli, O. Moutanabbir, S. Cecchi, G. Isella, T. Gemming, S.

Baunack, G. Chen, O. G. Schmidt, and A. Rastelli

Citation: Journal of Applied Physics 115, 044312 (2014); doi: 10.1063/1.4863115

View online: http://dx.doi.org/10.1063/1.4863115

View Table of Contents: http://scitation.aip.org/content/aip/journal/jap/115/4?ver=pdfcov

Published by the AIP Publishing

\section{Articles you may be interested in}

Dimensionality and heat transport in Si-Ge superlattices

Appl. Phys. Lett. 102, 073113 (2013); 10.1063/1.4792748

Analytical expression for thermal conductivity of superlattices

J. Appl. Phys. 107, 084303 (2010); 10.1063/1.3386464

Role of heat flow direction, monolayer film thickness, and periodicity in controlling thermal conductivity of a Si-Ge superlattice system

J. Appl. Phys. 105, 013541 (2009); 10.1063/1.3056135

Effect of nanodot areal density and period on thermal conductivity in Si Ge Si nanodot superlattices

Appl. Phys. Lett. 92, 053112 (2008); 10.1063/1.2842388

Thermal conductivity of Si/SiGe and SiGe/SiGe superlattices

Appl. Phys. Lett. 80, 1737 (2002); 10.1063/1.1455693

High-Voltage Amplifiers

-Voltage Range from $\pm 50 \mathrm{~V}$ to $\pm 60 \mathrm{kV}$

- Current to 25A

Electrostatic Voltmeters

- Contacting \& Non-contacting

- Sensitive to $1 \mathrm{mV}$

- Measure to $20 \mathrm{kV}$
ENABLING RESEARCH AND

INNOVATION IN DIELECTRICS,

ELECTROSTATICS,

MATERIALS, PLASMAS AND PIEZOS

Rek www.trekinc.com

TREK, INC. 190 Walnut Street, Lockport, NY 14094 USA •Toll Free in USA 1-800-FOR-TREK• (t):716-438-7555 • (f):716-201-1804 • sales@trekinc.com 


\title{
Thermal transport through short-period SiGe nanodot superlattices
}

\author{
Peixuan Chen, ${ }^{1,2, a)}$ J. J. Zhang, ${ }^{1,2}$ J. P. Feser, ${ }^{3}$ F. Pezzoli, ${ }^{2, b)}$ O. Moutanabbir, ${ }^{4}$ S. Cecchi, ${ }^{5}$ \\ G. Isella, ${ }^{5}$ T. Gemming, ${ }^{6}$ S. Baunack, ${ }^{2}$ G. Chen, ${ }^{1,7}$ O. G. Schmidt, ${ }^{2,8}$ and A. Rastelli ${ }^{1,2}$ \\ ${ }^{1}$ Institute of Semiconductor and Solid State Physics, Johannes Kepler University Linz, Altenbergerstr. 69 , \\ A-4040 Linz, Austria \\ ${ }^{2}$ Institute for Integrative Nanosciences, IFW Dresden, Helmholtzstr. 20, 01069 Dresden, Germany \\ ${ }^{3}$ Department of Materials Science and Engineering, Frederick-Seitz Materials Research Laboratory, \\ University of Illinois at Urbana-Champaign, Urbana, Illinois 61801, USA \\ ${ }^{4}$ Department of Engineering Physics, ÉcolePolytechnique de Montréal, Montréal, CP 6079, \\ Succursale Centre-Ville, Montréal, Québec H3C 3A7, Canada \\ ${ }^{5}$ L-NESS Dipartimento di Fisica-Politecnico di Milano, Polo Territoriale di Como, Via Anzani 42, \\ 22100 Como, Italy \\ ${ }^{6}$ Institute for Complex Materials, IFW Dresden, Helmholtzstr. 20, 01069 Dresden, Germany \\ ${ }^{7}$ State Key Laboratory of Infrared Physics, Shanghai Institute of Technical Physics, \\ Chinese Academy of Sciences, Shanghai 200083, People's Republic of China \\ ${ }^{8}$ Center for Advancing Electronics Dresden, TU Dresden, 01062 Dresden, Germany
}

(Received 12 November 2013; accepted 11 January 2014; published online 30 January 2014)

\begin{abstract}
The cross-plane thermal conductivity $\kappa$ of multilayers of SiGe nanodots separated either by Si or SiGe can be decreased by reducing the period length or by increasing the nanodot density. It is, however, not clear how far $\kappa$ can be reduced by using these strategies. In addition, the role of SiGe nanodots on the reduction of $\kappa$ is still not fully understood. In this work, we addressed these issues by studying experimentally the cross-plane $\kappa$ of $\mathrm{Ge} / \mathrm{Si}$ superlattices with period lengths down to $1.5 \mathrm{~nm}$. Although $\kappa$ tends to preserve the decreasing trend with reducing the period length, for periods shorter than $2 \mathrm{~nm}$ we observed a drastic drop of the average thermal resistance per period. This finding indicates a weakening of the effect of the interfaces on phonon scattering and implies a lower limit for $\kappa$. To assess the role played by the nanodots in the reduction of $\kappa$ we studied $\mathrm{Ge} / \mathrm{Si}$ superlattices with nanodot densities varying from 0 to $\sim 8 \times 10^{10} \mathrm{~cm}^{-2}$ and a fixed Si spacer thickness of $2.7 \mathrm{~nm}$. The experimental results suggest that $\mathrm{SiGe}$ nanodots with "pyramid"'-shape have an effect comparable to nominally planar wetting layers on the cross-plane thermal transport. Finally, the comparison of superlattices with nanodots separated by $\mathrm{Si}_{1-\mathrm{x}} \mathrm{Ge}_{\mathrm{x}}$ (with $\mathrm{x}$ from 0 to 0.2 ) shows that spacer alloying is beneficial in reducing the $\kappa$ by $\sim 20 \%$. The results presented in this work are expected to be relevant to micro/nanoscale energy conversion which requires minimizing the thermal conductivity of superlattice-based thin film thermoelectrics. (C) 2014 AIP Publishing LLC. [http://dx.doi.org/10.1063/1.4863115]
\end{abstract}

\section{INTRODUCTION}

The ability of thermoelectric materials to convert heat into electricity and vice versa has attracted great interest from scientists and engineers since the 1950 's. ${ }^{1}$ Despite several successful applications, ${ }^{2}$ thermoelectricity still remains confined to niche applications due to the low energy conversion efficiency of conventional materials. ${ }^{2}$ The energy conversion efficiency is related to the dimensionless figure of merit of $Z T$, which is proportional to the square of the Seebeck coefficient $S^{2}$, the electrical conductivity $\sigma$, the absolute temperature $T$, and inversely proportional to the thermal conductivity $\kappa^{3,4}$ Nanostructuring of thermoelectric materials has been widely employed in the last two decades with the aim to reduce the thermal conductivity and/or to increase the power factor $S^{2} \sigma,{ }^{1,5-7}$ thus enhancing the values of $Z T .^{8-13}$ Among different nanostructuring strategies, interfaces in superlattices or nanodots in a host matrix have emerged as promising pathways to

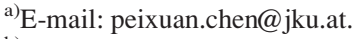

${ }^{\text {b) Present address: LNESS-Dipartimento di Scienza dei Materiali, Università }}$ degli Studi di Milano-Bicocca, via R. Cozzi 55, I-20125 Milano, Italy.
}

reduce the thermal conductivity through phonon scattering at multiple length-scales. ${ }^{14-18}$ Single-crystalline SiGe nanodot superlattices (SLs) are an ideal platform to study the effects of interfaces and nanodots on thermal transport not only because they consist of two elemental non-polar semiconductors, but also because the structural parameters, such as the interface spacing length (i.e., period length) and nanodot density can be precisely controlled during molecular beam epitaxial (MBE) growth. Reduction of cross-plane $\kappa$ with decreasing period length has been first reported by Lee et al. ${ }^{14}$ and Huxtable et al. ${ }^{15}$ on planar $\mathrm{Ge} / \mathrm{Si} \mathrm{SLs}$ and $\mathrm{Si}_{0.7} \mathrm{Ge}_{0.3} / \mathrm{Si} \mathrm{SLs}$, respectively. The minimum value of $\kappa$ was $\sim 3$ and $\sim 11 \mathrm{~W} / \mathrm{m} \cdot \mathrm{K}$ for period lengths of 3 and $7.5 \mathrm{~nm}$, respectively. ${ }^{14,15}$ Later on, even lower $\kappa$ values were found on SLs containing SiGe nanodots by Lee et al., ${ }^{17}$ who observed that $\kappa$ was reduced by decreasing the period length or by increasing the nanodot areal density. A minimum $\kappa$ of $\sim 2 \mathrm{~W} / \mathrm{m} \cdot \mathrm{K}$ was obtained for a $\mathrm{Ge} / \mathrm{Si} \mathrm{SL}$ with period length of $\sim 3.9 \mathrm{~nm}$ and nanodot density of $\sim 7 \cdot 10^{9} \mathrm{~cm}^{-2}$. More recently, a lower $\kappa$ of $\sim 1 \mathrm{~W} / \mathrm{m} \cdot \mathrm{K}$ was found by Pernot et al. ${ }^{18}$ on a SiGe nanodot superlattice (NDSL) with a similar period length of $\sim 3.7 \mathrm{~nm}$ but higher nanodot density reaching $\sim 5 \cdot 10^{10} \mathrm{~cm}^{-2}$. 
(a) $1^{\text {st }}$ series: $\mathrm{Ge} / \mathrm{Si}$ NDSLs on Si substrate variable period length

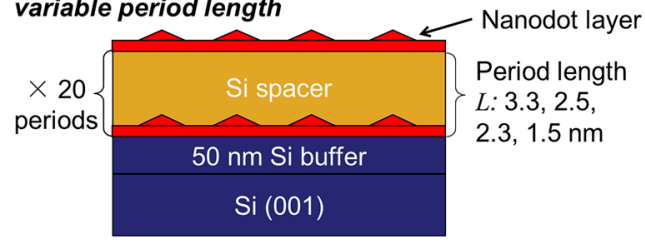

(c) $3^{\text {rd }}$ series: Ge/SiGe NDSLs on three different substrates, variable Ge concentration of SiGe spacer

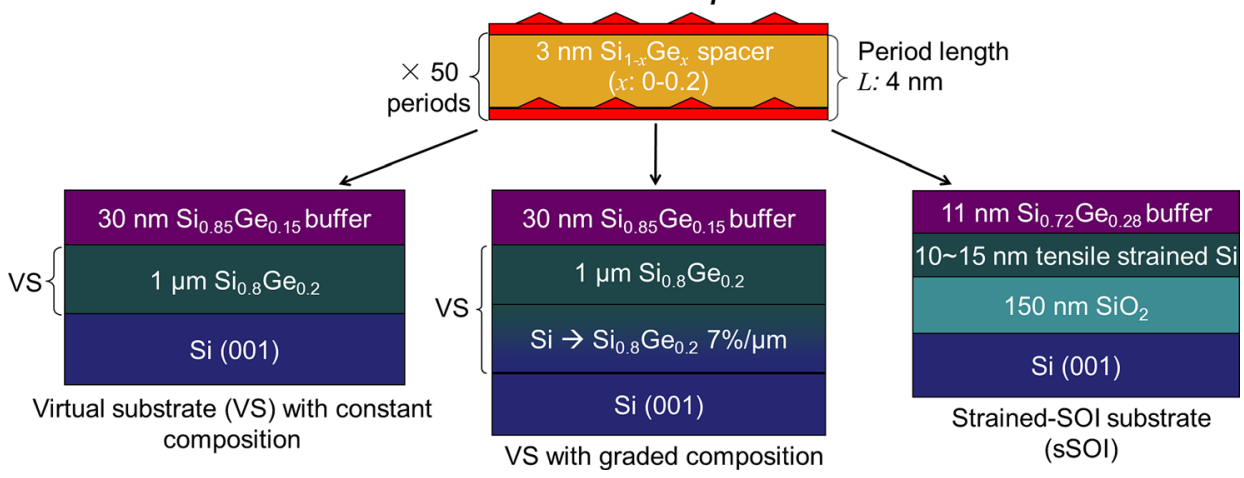

(b) $2^{\text {nd }}$ series: Ge/Si NDSLs on Si substrate,

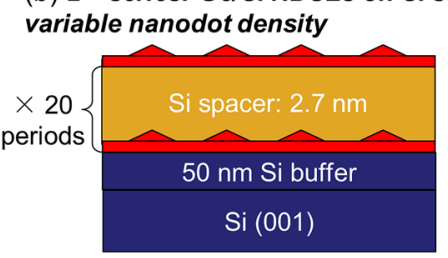

FIG. 1. Schematic illustration of the sample structures: (a) 1st sample series: $\mathrm{Ge} / \mathrm{Si}$ NDSLs grown on Si (001) substrate with variable period length from 3.3 to $1.5 \mathrm{~nm}$; (b) 2nd sample series: Ge/Si NDSLs grown on Si (001) substrate with variable nanodot density from 0 to $\sim 8 \times 10^{10} \mathrm{~cm}^{-2}$ and fixed $\mathrm{Si}$ spacer thickness of $2.7 \mathrm{~nm}$; (c) 3rd sample series: Ge/SiGe NDSLs grown on two kinds of virtual substrate (VS) and strained silicon-on-insulator (sSOI) substrate with constant $\mathrm{Si}_{1-\mathrm{x}} \mathrm{Ge}_{\mathrm{x}}$ spacer thickness of $3 \mathrm{~nm}$, Ge layer thicknesses of $1 \mathrm{~nm}$ and variable Ge concentration of the SiGe spacer layer from $0 \%$ to $20 \%$.
Despite previous investigations, which show that $\kappa$ can be lowered by shortening the period length ${ }^{14,15,17,18}$ and/or by increasing the nanodot areal density of SiGe SLs, ${ }^{17}$ the understanding of the effects produced by interfaces and nanodots on thermal transport is still not satisfactory. First, it is still not clear how far $\kappa$ can be reduced by shortening the period length. Recently, Garg and Chen ${ }^{19}$ theoretically predicted that the interplay between a decrease in phonon relaxation time and an increase in phonon group velocity with decreasing period length would lead to a minimum in the cross-plane $\kappa$ for Ge/Si SLs. According to this work, an increase of $\kappa$ with decreasing period length can be expected for period lengths below $3 \mathrm{~nm}$. However, most of the previous experimental works focused on SiGe SLs with period lengths larger than $3.5 \mathrm{~nm}$. Second, the role of SiGe nanodots on the reduction of $\kappa$ is still not fully understood. Kim and co-workers ${ }^{16}$ firstly demonstrated that the inclusion of 0.3 vol. \% of ErAs nanodots in $\operatorname{In}_{0.53} \mathrm{Ga}_{0.47}$ As alloy matrix was sufficient to reduce $\kappa$ by a factor of 2 . This was attributed to phonon scattering at multiple length-scales, with short wavelength phonons being mostly scattered by the alloy matrix and mid-to-long-wavelength phonons by the ErAs nanodots with diameters of a few nanometers. However, different from the ErAs nanodots in an InGaAs matrix, which grow according to the Volmer-Weber mode, the growth of SiGe nanodots on $\mathrm{Si}$ (001) follows the Stranski-Krastanow mode, i.e., SiGe nanodots form on top of a planar wetting layer (WL) to partially relax the strain due to the lattice mismatch $^{20}$ or to lower the surface energy. ${ }^{21,22}$ As a consequence, in the case of $\mathrm{SiGe}$ nanodots, it is difficult to discriminate the effects produced by nanodots and WL on the thermal transport.

In this paper, we first present details of the sample growth and of the $3 \omega$ method, which we use to determine the cross-plane $\kappa$ of SiGe NDSLs, with particular attention to the evaluation of measurement uncertainties. We then present the results of thermal conductivity measurements of SiGe NDSLs with period lengths in heretofore unexplored range from $3.3 \mathrm{~nm}$ to $1.5 \mathrm{~nm}$ (Refs. 17 and 18 focused on SLs with larger period lengths from $\sim 4$ to $\sim 20 \mathrm{~nm}$ ). We show that while $\kappa$ tends to preserve the decreasing trend with reducing the period length, a weakening of the interfaces' effect on the thermal transport is observed when the period length is shorter than $2 \mathrm{~nm}$. To elucidate the role of nanodots, we study the cross-plane $\kappa$ of SiGe NDSLs with nanodot densities varying from 0 to $\sim 8 \cdot 10^{10} \mathrm{~cm}^{-2}$ and fixed Si spacer thickness of $2.7 \mathrm{~nm}$. Our experimental results suggest that the effect of pyramid-shaped nanodots on the thermal transport is similar to that of planar WLs. Finally, we investigate the effect produced by replacing the $\mathrm{Si}$ spacer layers with $\mathrm{Si}_{1-\mathrm{x}} \mathrm{Ge}_{\mathrm{X}}$ layers and find that alloying leads to a slight reduction of $\kappa$.

\section{EXPERIMENTAL DETAILS}

\section{A. Sample growth}

The SiGe NDSLs used in this work were grown by solid-source $\mathrm{MBE}$ on (001) oriented substrates at a fixed temperature of $500{ }^{\circ} \mathrm{C}$. There are three sample series as schematically shown in Fig. 1. The first sample series is used to investigate the effect of period length on the cross-plane $\kappa$. The samples consist of Ge/Si NDSLs with a period length $L$ varying from 3.3 to $1.5 \mathrm{~nm}$ grown on $\mathrm{Si}(001)$, as illustrated in Fig. 1(a). The period length is the sum of the thickness of the Si spacer and the average deposited amount of Ge per nanodot layer $t_{G e}$. In order to limit the occurrence of plastically relaxed nanodots and maintain the nanodot density constant in the layers, the deposited amount of $\mathrm{Ge}$ in the upper nanodot layers was properly reduced as compared to the bottommost layer, following the approach in Ref. 23. There is a slight difference in the average deposited amount of Ge per nanodot layer between different NDSLs (see growth parameters in Table I).

The second sample series is used to investigate the effects of nanodots and WLs on the cross-plane $\kappa$. The samples consist of Ge/Si NDSLs with constant Si spacer thickness of $2.7 \mathrm{~nm}$ grown on $\mathrm{Si}(001)$. The $\mathrm{SiGe}$ nanodot density 
TABLE I. Sample growth protocols for all three series as illustrated in Fig. 1. VS: virtual substrate. sSOI: strained silicon-on-insulator substrate. The substrate temperature during growth is $500{ }^{\circ} \mathrm{C}$.

\begin{tabular}{|c|c|c|c|c|c|c|c|c|}
\hline $\begin{array}{l}\text { Sample } \\
\text { No. }\end{array}$ & $\begin{array}{l}\text { Sample } \\
\text { series } \\
\text { No. }\end{array}$ & Substrate & $\begin{array}{c}\text { Ge amount of each period } \\
\left(t_{G e}\right), \text { starting from } \\
\text { bottommost layer }(\AA)\end{array}$ & $\begin{array}{c}\text { Thickness of } \\
\mathrm{Si}_{1-\mathrm{x}} \mathrm{Ge}_{\mathrm{x}} \text { spacer } \\
(\mathrm{nm})\end{array}$ & $\begin{array}{l}\text { Ge fraction } \\
\text { of } \mathrm{Si}_{1-\mathrm{x}} \mathrm{Ge}_{\mathrm{x}} \\
\text { spacer }\end{array}$ & $\begin{array}{l}\text { Average period } \\
\text { length }(\mathrm{nm})\end{array}$ & $\begin{array}{l}\text { Total Ge amount } \\
\text { in SL layer (nm) }\end{array}$ & $\begin{array}{l}\text { Nanodot density } \\
\text { on topmost layer } \\
\quad\left(10^{10} \mathrm{~cm}^{-2}\right)\end{array}$ \\
\hline 1 & 1 st & $\mathrm{Si}(001)$ & $8.4,6.7,6.0,5.7,17 \times(5.4)$ & 2.7 & 0 & 3.3 & 11.9 & 7.6 \\
\hline 2 & 1 st & $\mathrm{Si}(001)$ & $8.4,5.9,4.9,4.2,17 \times(3.9)$ & 2.1 & 0 & 2.5 & 9.0 & 5.8 \\
\hline 3 & 1 st & $\mathrm{Si}(001)$ & $8.4,6.2,5.3,4.8,17 \times(4.3)$ & 1.9 & 0 & 2.3 & 9.8 & 9.0 \\
\hline 4 & 1 st & $\mathrm{Si}(001)$ & $8.4,5.9,4.9,4.3,17 \times(4.1)$ & 1.0 & 0 & 1.5 & 9.3 & 11.0 \\
\hline 5 & 2nd & $\mathrm{Si}(001)$ & $7.8,6.2,5.6,5.3,17 \times(5)$ & 2.7 & 0 & 3.2 & 11.0 & 8.3 \\
\hline 6 & 2 nd & $\mathrm{Si}(001)$ & $6.9,5.5,4.9,6.4,17 \times(4.4)$ & 2.7 & 0 & 3.2 & 9.7 & 4.5 \\
\hline 7 & 2 nd & $\mathrm{Si}(001)$ & $7.2,5.4,4.7,4.4,17 \times(3.6)$ & 2.7 & 0 & 3.1 & 8.3 & 1.1 \\
\hline 8 & $2 \mathrm{nd}$ & $\mathrm{Si}(001)$ & $5.6,4.2,3.7,3.4,17 \times(2.8)$ & 2.7 & 0 & 3.0 & 6.5 & 0 \\
\hline 9 & $3 \mathrm{rd}$ & Constant VS & $51 \times(9.8)$ & 3 & 0 & 4 & 50.0 & 1.5 \\
\hline 10 & $3 r d$ & Constant VS & $51 \times(9.8)$ & 3 & 0.09 & 4 & 63.0 & 1.6 \\
\hline 11 & $3 r d$ & Constant VS & $51 \times(9.8)$ & 3 & 0.15 & 4 & 72.5 & 1.0 \\
\hline 12 & $3 r d$ & Constant VS & $51 \times(9.8)$ & 3 & 0.20 & 4 & 80.0 & 1.1 \\
\hline 13 & $3 r d$ & Graded VS & $51 \times(9.8)$ & 3 & 0 & 4 & 50.0 & 1.4 \\
\hline 14 & $3 \mathrm{rd}$ & Graded VS & $51 \times(9.8)$ & 3 & 0.09 & 4 & 63.0 & 1.7 \\
\hline 15 & $3 r d$ & Graded VS & $51 \times(9.8)$ & 3 & 0.15 & 4 & 72.5 & 0.8 \\
\hline 16 & $3 r d$ & Graded VS & $51 \times(9.8)$ & 3 & 0.20 & 4 & 80.0 & 1.0 \\
\hline 17 & $3 r d$ & sSOI & $51 \times(9.8)$ & 3 & 0 & 4 & 50.0 & 1.6 \\
\hline 18 & $3 \mathrm{rd}$ & sSOI & $51 \times(9.8)$ & 3 & 0.09 & 4 & 63.0 & 1.8 \\
\hline 19 & $3 r d$ & sSOI & $51 \times(9.8)$ & 3 & 0.15 & 4 & 72.5 & 2.1 \\
\hline 20 & $3 r d$ & sSOI & $51 \times(9.8)$ & 3 & 0.20 & 4 & 80.0 & 1.8 \\
\hline
\end{tabular}

varies from 0 to $\sim 8 \times 10^{10} \mathrm{~cm}^{-2}$ by gradually increasing the average $t_{G e}$ in the nanodot layer, as illustrated in Fig. 1(b). We will show that by comparing $\kappa$ 's of SLs with and without nanodots, and SLs with variable nanodot density, the effects of nanodots and WLs on the thermal transport can be discriminated.

The third sample series is used to investigate the effect of alloying the matrix on the cross-plane $\kappa$ of the $\mathrm{Ge} / \mathrm{SiGe}$ NDSLs. The samples in this series consist of $\mathrm{Ge} / \mathrm{SiGe}$ NDSLs with variable Ge concentration of the SiGe layer (see Fig. 1(c)). The thicknesses of the SiGe spacers and $t_{G e}$ for the nanodot layer are fixed. Since Ge/SiGe NDSLs have larger average $\mathrm{Ge}$ concentration as compared to the $\mathrm{Ge} / \mathrm{Si}$ NDSLs of the first two sample series, high density of defects may be expected if the NDSLs are grown on $\mathrm{Si}(001)$ substrates due to the lattice mismatch between the NDSLs and the substrate. In order to minimize the occurrence of defects, we grew the Ge/SiGe NDSLs on SiGe virtual substrates and strained silicon-on-insulator (sSOI) substrates. The virtual substrates were obtained by low-energy plasma-enhanced chemical vapour deposition (LEPECVD). ${ }^{24}$ Two kinds of virtual substrate (VS) were used (see Fig. 1(c)). In both cases, a thick SiGe buffer was grown on conventional $\mathrm{Si}(001)$ substrates with the final target of obtaining strainrelaxed $\mathrm{Si}_{0.8} \mathrm{Ge}_{0.2}$. The first VS (the left one in Fig. 1(c)) consists of a $1 \mu \mathrm{m}$-thick SiGe layer with constant composition. The second VS (the middle one in Fig. 1(c)) features a compositionally graded buffer followed by a constant composition part with a total thickness of $\sim 4 \mu \mathrm{m}$. The grading rate was $7 \% / \mu \mathrm{m}$ and the constant composition layer is $1 \mu \mathrm{m}$ thick. The sSOI substrates were obtained using direct wafer bonding and an ion-cut process. ${ }^{25} \mathrm{~A} \sim 10-15 \mathrm{~nm}$-thick strained $\mathrm{Si}$ layer was first grown on a relaxed $\mathrm{Si}_{0.7} \mathrm{Ge}_{0.3}$, transferred onto a $\mathrm{Si}$ handle substrate capped with a $\sim 150 \mathrm{~nm}$ thick $\mathrm{SiO}_{2}$ layer. ${ }^{26}$ The transferred Si layer is under $1.2 \%$-tensile biaxial strain as expected from the growth on relaxed $\mathrm{Si}_{0.7} \mathrm{Ge}_{0.3}$. Accordingly, the lattice mismatch between Ge/SiGe NDSLs and the VS and the sSOI substrates was reduced. The actual SL thicknesses of selected samples were quantified by transmission electron microscopy. The surface morphology of all samples was characterized by atomic force microscopy (AFM). A detailed description of the growth parameters of all NDSLs used in this work is reported in Table I.

\section{B. Thermal conductivity measurement with the differential $3 \omega$ method}

\section{Measurement principle}

The cross-plane $\kappa$ of most of the SLs in this work was measured with the differential $3 \omega$ method $^{27,28}$ at room temperature. $3 \omega$ measurements were performed with instrumentation and conditions similar to those described in Ref. 18. In this method, a metal strip of about $6 \mu \mathrm{m}$ or $10 \mu \mathrm{m}$ width is used both to locally heat the sample structure (via an AC current with angular frequency $\omega$ ) and to monitor the local temperature rise $\Delta T$ on sample surface (or the strip) by detecting the voltage drop at angular frequency $3 \omega .^{27,28}$ To avoid current leakage through the substrate, an $\mathrm{Al}_{2} \mathrm{O}_{3}$ insulating layer is deposited by atomic layer deposition (ALD) on the samples prior to metal strip fabrication. The strip width is always more than one order of magnitude larger than the thickness of the SiGe SL and the $\mathrm{Al}_{2} \mathrm{O}_{3}$ layer, leading to negligible lateral heat spreading effects in the SL and the $\mathrm{Al}_{2} \mathrm{O}_{3}$ layer. ${ }^{29,30}$ Thus, a simple one-dimensional heat conduction analysis is applicable to determine the $\kappa$ of the SL. ${ }^{28,31}$ Since the temperature rise $\Delta T$ on the strip is the sum 
(a)

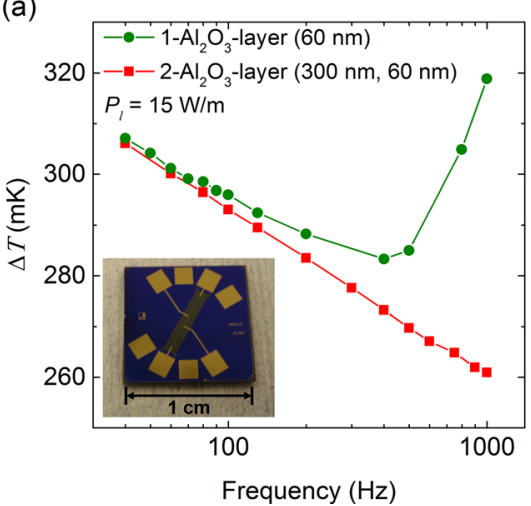

(b)

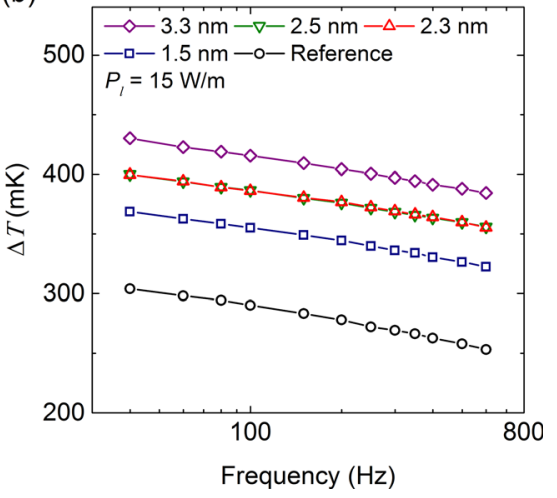

FIG. 2. (a) $3 \omega$ raw data for Si samples with a single insulating layer (1$\mathrm{Al}_{2} \mathrm{O}_{3}$-layer) and double insulating layer (2- $\mathrm{Al}_{2} \mathrm{O}_{3}$-layer); (b) $3 \omega$ raw data for NDSL samples with variable period length (sample series 1 in Fig. 1(a)) and for a reference sample without NDSL. The inset in (a) shows an image of one of the studied samples. of the temperature rise across the Si substrate, the SL layer and the $\mathrm{Al}_{2} \mathrm{O}_{3}$ layer, the temperature rise across the SL layer can be obtained by comparing the values of $\Delta T$ for the samples with SLs and for a reference sample without SL. To limit systematic errors the $\mathrm{Al}_{2} \mathrm{O}_{3}$ layer was deposited on SL and reference samples simultaneously. To account for fluctuations in the width of the metal strip deposited on different samples, we have measured all strips by AFM.

The analytical expression for the real part of the temperature rise in the reference samples, $\Delta T_{1}$, is ${ }^{28,29}$

$$
\begin{aligned}
\Delta T_{1} & =\frac{P_{l}}{\pi \kappa_{S i}}\left\{\frac{1}{2} \ln \left(\frac{\kappa_{S i}}{C_{S i} \rho_{S i} b_{r e f}^{2}}\right)+\eta_{C}-\frac{1}{2} \ln (2 \omega)\right\}+\frac{P_{l}}{2 b_{r e f}} R_{D}, \\
& =\Delta T_{\text {sub }}\left(b_{r e f}, \omega\right)+\frac{P_{l}}{2 b_{r e f}} R_{D},
\end{aligned}
$$

where $P_{l}$ is the electric power per unit length applied on the strip, $2 b_{r e f}$ is the width of the metal strip for the reference sample, $\eta_{C}$ is a constant. Here $\kappa_{S i}, C_{S i}$, and $\rho_{S i}$ are the thermal conductivity, specific heat, and mass density of the $\mathrm{Si}$ substrate, respectively. ${ }^{32}$ The first term in Eq. (1) is the frequency dependent temperature rise across the $\mathrm{Si}$ substrate, and the second term is the temperature rise across the $\mathrm{Al}_{2} \mathrm{O}_{3}$ layer. The cross-plane heat transport in the dielectric layer adds a frequency-independent component to the total thermal response as described by the thermal resistance $R_{D}\left(R_{D}\right.$ is the sum of thermal resistances of the dielectric layer, interface thermal resistances between $\mathrm{Si}$ and dielectric layer and between dielectric layer and metal strip). Likewise, the temperature rise measured on the strip placed on the NDSL sample, $\Delta T_{2}$ can be written as

$$
\begin{aligned}
\Delta T_{2}= & \frac{P_{l}}{\pi \kappa_{S i}}\left\{\frac{1}{2} \ln \left(\frac{\kappa_{S i}}{C_{S i} \rho_{S i} b_{S L}^{2}}\right)+\eta_{C}-\frac{1}{2} \ln (2 \omega)\right\} \\
& +\frac{P_{l}}{2 b_{S L}}\left(R_{D}+R_{S L}\right), \\
= & \Delta T_{\text {sub }}^{\prime}\left(b_{S L}, \omega\right)+\frac{P_{l}}{2 b_{S L}}\left(R_{D}+\frac{t_{S L}}{\kappa}\right),
\end{aligned}
$$

where $2 b_{S L}$ is the strip width for the NDSL sample, $R_{S L}, t_{S L}$ and $\kappa$ are thermal resistance, thickness and thermal conductivity of the SL layer, respectively. Since the $\mathrm{Al}_{2} \mathrm{O}_{3}$ layer was deposited on the SL samples and the reference sample during the same deposition run, the thermal resistance $R_{D}$ can be assumed as a constant. The thermal conductivity of the NDSL can be therefore determined from Eqs. (1) and (2) as follows:

$$
\kappa=\frac{P_{l}}{2 b_{S L}} \cdot \frac{t_{S L}}{\left[\Delta T_{2}-\Delta T_{\text {sub }}^{\prime}-\frac{b_{r e f}}{b_{S L}}\left(\Delta T_{1}-\Delta T_{\text {sub }}\right)\right]} .
$$

\section{Dielectric layer with reduced thermal resistance: Two-layer method}

According to Eq. (2), the parasitic $R_{D}$ of the dielectric layer contributes to the background for the measurement of $\kappa$ of the SL layer (the rest of the background comes from the $\mathrm{Si}$ substrate). The precision of the measurement of a thin film, such as the NDSL here, can be improved by scaling down the thickness of the $\mathrm{Al}_{2} \mathrm{O}_{3}$ layer, so that $R_{D}$ is lower or comparable to $R_{S L}$. The measured $R_{D}$ of a $60 \mathrm{~nm}$-thick $\mathrm{Al}_{2} \mathrm{O}_{3}$ layer and interfaces is $\sim 4 \cdot 10^{-8} \mathrm{~m}^{2} \cdot \mathrm{K} / \mathrm{W}$, which is comparable to the calculated $R_{S L}$ of most SL layers in this work. However, reducing the thickness of $\mathrm{Al}_{2} \mathrm{O}_{3}$ also deteriorates its insulating quality, thus affecting the $3 \omega$ measurement. According to Eq. (1), the measured $\Delta T(\omega)$ is expected to be linearly proportional to $-\ln (\omega)$. However, the measurement on a $\mathrm{Si}$ sample covered with only $60 \mathrm{~nm}$-thick $\mathrm{Al}_{2} \mathrm{O}_{3}$ deviates from this behavior at relatively high frequencies $(400-1000 \mathrm{~Hz})$, see circle-line in Fig. 2(a). This may be ascribed to current leakage through the thin $\mathrm{Al}_{2} \mathrm{O}_{3}$ layer, as the measurement on a Si sample covered with $300 \mathrm{~nm}$-thick $\mathrm{Al}_{2} \mathrm{O}_{3}$ does not show such an anomalous behavior (not shown here). In order to minimize the current leakage while keeping the $\mathrm{Al}_{2} \mathrm{O}_{3}$ as thin as possible, we used a two-layermethod for the dielectric film: ${ }^{33}$ The strip heater is placed on top of a $60 \mathrm{~nm}$-thick $\mathrm{Al}_{2} \mathrm{O}_{3}$ layer, while the contact pads are

TABLE II. Relative uncertainties and corresponding distribution function for the experimentally measured parameters entering into Eq. (3). These are used to obtain the uncertainties on the thermal conductivity of the SLs via error propagation accomplished through a Monte Carlo calculation.

\begin{tabular}{ccccc}
\hline \hline & $\begin{array}{c}\text { Thickness }\left(t_{S L}\right) \\
\text { uncertainty } \\
(\%)\end{array}$ & $\begin{array}{c}\text { Strip width }(b) \\
\text { uncertainty } \\
(\mu \mathrm{m})\end{array}$ & $\begin{array}{c}\text { Power } \\
\left(P_{l}\right) \\
\text { uncertainty } \\
(\%)\end{array}$ & $\begin{array}{c}\text { Temperature } \\
(\Delta T)\end{array}$ \\
$\begin{array}{c}5 \\
\text { uncertainty } \\
(\%)\end{array}$ \\
\hline \hline
\end{tabular}


(a)

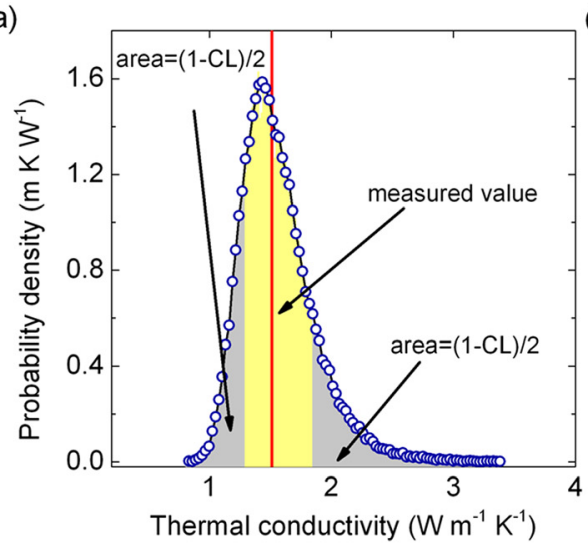

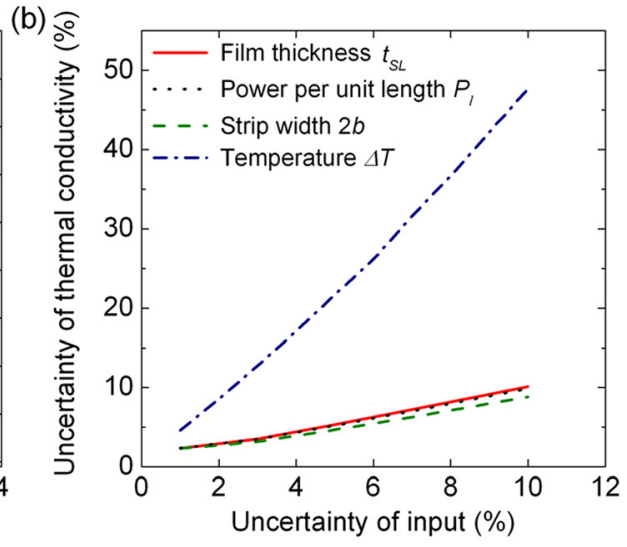

FIG. 3. (a) Probability density distribution of the calculated thermal conductivity for a sample with $2.7 \mathrm{~nm}$-thick Si spacer and nanodot density of $7.6 \times 10^{10} \mathrm{~cm}^{-2}$. The probability density is obtained by Monte Carlo calculation using Eq. (3) and the experimentally measured quantities with their uncertainties. The yellow area around the measured value (red line) contains in total the $68.3 \%$ (confident level, CL) of the counts of the histogram. (b) Calculated relative uncertainty on measured thermal conductivity for the same sample used in (a) by varying the uncertainty of one of the inputs into Eq. (3) while keeping the uncertainties of other parameters as zero.

fabricated on top of a $360 \mathrm{~nm}$-thick $\mathrm{Al}_{2} \mathrm{O}_{3}$ layer. The inset in Fig. 2(a) shows a picture of one of the studied samples with size of $1 \mathrm{~cm} \times 1 \mathrm{~cm}$. The purple color corresponds to the area with $360 \mathrm{~nm}$-thick $\mathrm{Al}_{2} \mathrm{O}_{3}$ and the brown color in the center corresponds to the area with only $60 \mathrm{~nm}$-thick $\mathrm{Al}_{2} \mathrm{O}_{3}$. The square-line in Fig. 2(a) is the measurement on a $\mathrm{Si}$ sample with two $\mathrm{Al}_{2} \mathrm{O}_{3}$ layers $(300 \mathrm{~nm}$ and $60 \mathrm{~nm})$. We can see that $\Delta T(\omega)$ is linearly proportional to $-\ln (\omega)$ in the frequency range between $40 \mathrm{~Hz}$ and $1000 \mathrm{~Hz}$. Similar results were obtained on all other samples investigated so far. Thus, the two-layer-method can improve the measurement precision by allowing a reduction of the thickness of the dielectric film while avoiding current leakage. Fig. 2(b) shows $3 \omega$ raw data on NDSLs with variable period length (sample series 1, see Fig. 1(a)) and a reference sample without NDSL. In spite of the limited NDSL layer thickness, between 30 and $70 \mathrm{~nm}$, a clear temperature rise on the NDSL layer is visible, reproducible, and large enough for calculating the thermal conductivity according to Eqs. (1)-(3).

\section{Monte Carlo calculations of the measurement uncertainty}

The calculation of $\kappa$ according to Eq. (3) depends on several experimentally measured quantities (with their own uncertainties) in a nonlinear fashion. Furthermore, because uncertainties are relatively large when measuring very thin films, common error propagation approaches are no longer accurate. To address this issue, we performed a simple Monte Carlo calculation to propagate the uncertainties. In this approach, we randomly generated all the quantities entering Eqs. (1)-(3) according to their measured values, uncertainties, and expected statistical distributions (see Table II). From each set of random values we obtained, from Eq. (3), the corresponding value of $\kappa$. By iterating the calculation step many times (here 20000 for each value of frequency) and by plotting the results in form of a histogram, we obtained a probability density distribution for $\kappa$.

As an example, Fig. 3(a) shows the probability density for an NDSL with Si spacer of $2.7 \mathrm{~nm}$, nanodot density of
$7.6 \times 10^{10} \mathrm{~cm}^{-2}$ and period number of 20 . As expected, the calculated probability density distribution of $\kappa$ does not follow a normal distribution and is slightly asymmetric. To calculate the uncertainty of the measured $\kappa$, we defined a confidence interval containing $68.3 \%$ (confidence level, CL) of the events around the measured value, as indicated by the yellow region in Fig. 3(a). The uncertainty of the measured $\kappa$ is obtained from the difference between the values which define the boundary of the yellow area in Fig. 3(a) and the measured value.

To understand how the uncertainty on each parameter influences the uncertainty on $\kappa$, we vary the uncertainty of one of the input quantities while keeping those of others as zero. The result is shown in Fig. 3(b), with the plotted uncertainties being the average value of the lower and upper errors. From the calculation results, we find that minimizing the uncertainty of the measured temperature oscillations on the metal strip is the most efficient way to reduce the overall uncertainty of the measured $\kappa$ of the SL layer. Therefore, a careful calibration of the variation of the strip electrical resistance with temperature is essential. Further reduction of the measurement uncertainty might be possible if the Au strip is replaced by a metal with higher temperature coefficient of resistance, such as $\mathrm{Ni}$ (with temperature coefficient $\sim 1.6$ times larger than for $\mathrm{Au}$ ). Another way is to increase the signal/background ratio by increasing the thickness or the total thermal resistance of the thin film.

\section{RESULTS AND DISCUSSIONS}

\section{A. Effect of ultra-short period length on the thermal conductivity}

In this section, we discuss the effect of the period length on the cross-plane $\kappa$ of NDSLs. Figs. 4(a)-4(d) show the surface morphology of as-grown NDSLs with variable period length between 1.5 and $3.3 \mathrm{~nm}$. The insets are crosssectional TEM images of the NDSLs. The values of the actual period length $(L)$ are obtained from the TEM measurements. Fig. 5(a) shows the measured $\kappa$ as a function of the period length. When the period length is decreased from 

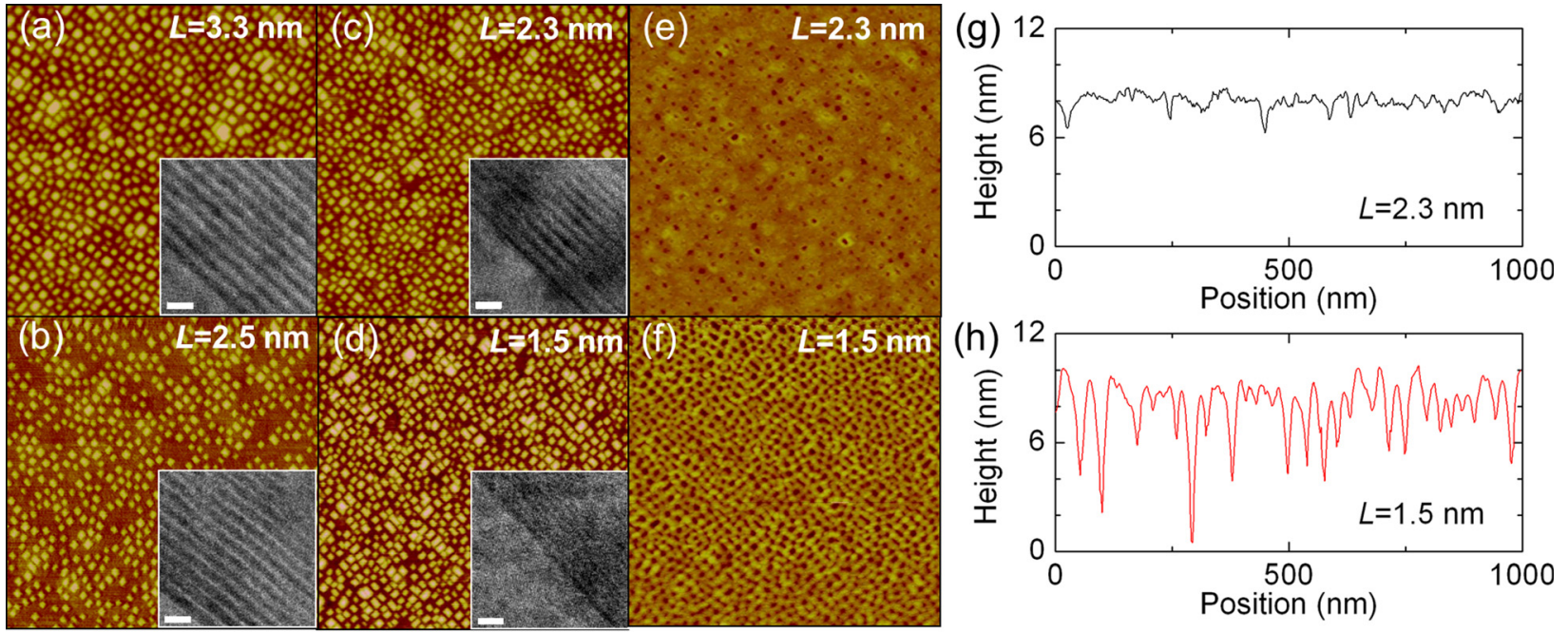

$1 \mu \mathrm{m} \times 1 \mu \mathrm{m}[110]$

$4 \mathrm{~nm}$

$10 \mathrm{~nm}$

FIG. 4. AFM images of as-grown NDSLs of sample series 1 with period length $L$ of (a) $3.3 \mathrm{~nm}$, (b) $2.5 \mathrm{~nm}$, (c) $2.3 \mathrm{~nm}$, and (d) $1.5 \mathrm{~nm}$, respectively. Insets in (a)-(d) are corresponding cross-sectional TEM images. The white scale bar corresponds to $5 \mathrm{~nm}$. AFM images for the sample with $L=2.3$ (e) and $1.5 \mathrm{~nm}$ (f) after $1 \mathrm{~h}$ etching in NHH solution. The height scale bar $0-4 \mathrm{~nm}$ refers to as-grown samples (a)-(d), whereas the scale bar $0-10 \mathrm{~nm}$ refers to etched samples (e) and (f). Representative AFM linescans of etched samples with period length of ( $\mathrm{g}$ ) $2.3 \mathrm{~nm}$ and (h) $1.5 \mathrm{~nm}$ along the [110] direction, respectively.

3.3 to $1.5 \mathrm{~nm}, \kappa$ slightly varies from $1.5 \pm 0.2 \mathrm{~W} / \mathrm{m} \cdot \mathrm{K}$ to 1.2 $\pm 0.2 \mathrm{~W} / \mathrm{m} \cdot \mathrm{K}$. The reproducibility of the low thermal conductivity values achieved in nanostructured SiGe-based superlattices is corroborated by the comparison in Fig. 5(b) of our new measurements with the previous report on NDSLs from Ref. 18, which spans larger period lengths, (a)

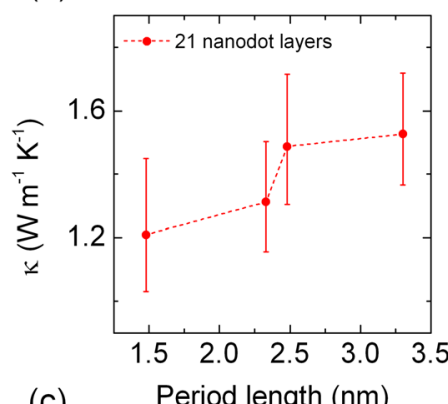

(c)

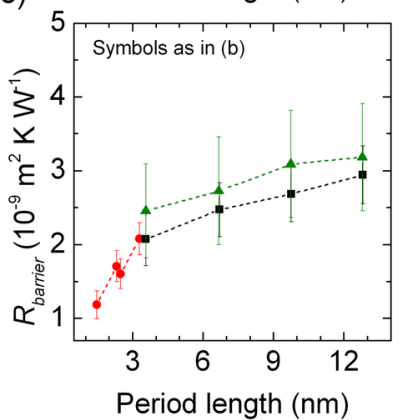

(b)

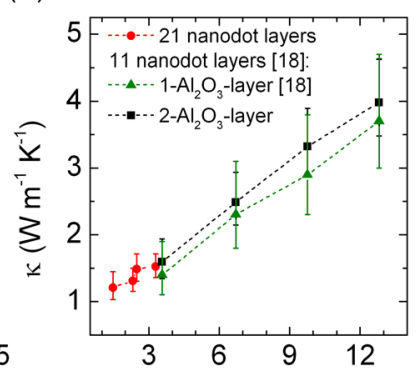

(d)

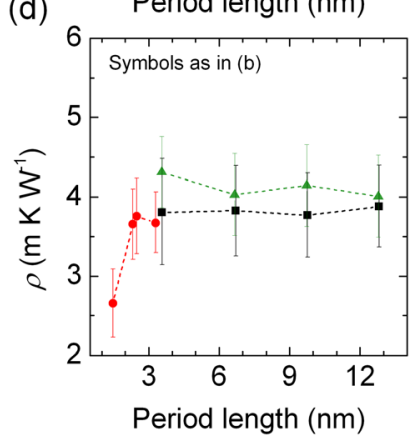

FIG. 5. (a) and (b) Thermal conductivity $\kappa$, (c) barrier thermal resistance $R_{\text {barrier }}$ and (d) thermal resistivity $\rho$ of NDSLs of sample series 1 (red circles) as a function of the period length, together with values from samples used in Ref. 18 (see black squares and green triangles in (b)-(d), which refer to SLs with 11 Ge nanodot layers). The data in Ref. 18 were measured with the $1-\mathrm{Al}_{2} \mathrm{O}_{3}$-layer method and are shown as green triangles in (b)-(d), while measurements on the same samples with the $2-\mathrm{Al}_{2} \mathrm{O}_{3}$-layer method are shown as black squares in (b)-(d). i.e., from $3.3 \mathrm{~nm}$ to $12.8 \mathrm{~nm}$ (triangles in Fig. 5(b)). Besides the good agreement between these two sets of data, we point out that our $3 \omega$ measurements are expected to have an improved accuracy since any possible current leakage has been minimized by using the 2-layer-method for the dielectric coating as discussed in Sec. II B 2. We also repeated the $3 \omega$ measurements on the same samples used in Ref. 18 by using the 2-layer-method for the dielectric layer. Although the new results are compatible with the previous ones within the uncertainties, the new measurements (squares in Fig. 5(b)) show an improved agreement with the measurements on our SLs, i.e., the small gap seen in Fig. 5(b) disappears. Noteworthy, Fig. 5(b) shows that $\kappa$ tends to preserve the decreasing trend with reducing the period length below $3 \mathrm{~nm}$. Reference 19 has recently predicted a minimum $\kappa$ with decreasing period length. The prediction was based on the interplay between increasing group velocity and decreasing phonon relaxation time with decreasing period length. However, we do not observe such a minimum value within our experimental accessible range of the period length.

As proposed in Ref. 18, the thermal resistance of a NDSL $R_{S L}$ is the sum of the barrier thermal resistance $\left(R_{\text {barrier }}\right)$ of the nanodot layers because of quasiballistic transport of phonons in the Si spacer followed by diffusive scattering at the Ge nanodot layers. Accordingly, we compare the average $R_{\text {barrier }}$ of our NDSLs with the results in Ref. 18 by normalizing $R_{S L}$ with the number of nanodot layer $(N)$. Fig. 5(c) shows the calculated $R_{\text {barrier }}$ as a function of the period length. $R_{\text {barrier }}$ slightly decreases when the period length decreases from 12.8 to $3.3 \mathrm{~nm}$. This may be attributed to the different Ge amount $t_{G e}$ used in the NDSLs. ${ }^{18} \mathrm{~A}$ constant "thermal resistivity" $\rho$ is obtained after normalizing the total thermal resistance with the total Ge amount in the SL (see triangles and squares in Fig. 5(d)). However, a drastic drop of $R_{\text {barrier }}$ with further decreasing the period length below $3.3 \mathrm{~nm}$ is observed for our NDSLs, as shown in 
Fig. 5(c). In order to exclude the influence of the different Ge amounts in the SLs (see Table I), we then compare the $\rho$ values of all NDSLs (see Fig. 5(d)). We find that while $\rho$ is almost constant when the period length is larger than $2 \mathrm{~nm}$, it drops when the period length is shorter than $2 \mathrm{~nm}$. Indeed, we decrease the period length mostly by reducing the thickness of the Si spacer, as the Ge layer thickness is in most of the samples much smaller than that of Si. One can imagine that when the thickness of the $\mathrm{Si}$ spacers approaches zero, the interface effect on the phonon transport will diminish. In the extreme case that the Ge layers are connected together, $\kappa$ would then approach the value of a thin Ge layer. Because the total amount of Ge in our SLs is about $10 \mathrm{~nm}$, we then estimate the thermal conductivity of a $10 \mathrm{~nm}$-thick Ge layer by using a simple calculation method as proposed by Wang and Mingo. ${ }^{34} \mathrm{We}$ obtain a value of about $9 \mathrm{~W} / \mathrm{m} \cdot \mathrm{K}$, corresponding to $\rho$ of about $0.1 \mathrm{~m} \cdot \mathrm{K} / \mathrm{W}$. Consequently, the drop of $\rho$ for short period lengths is not surprising. A question is why the drop of $\rho$ occurs when the period length is shorter than $2 \mathrm{~nm}$.

There are different processes which may explain the drop of $\rho$. First, since the Ge surface energy is lower than the $\mathrm{Si}$ surface energy, when a Ge layer is overgrown with a $\mathrm{Si}$ layer, $\mathrm{Ge}$ atoms will diffuse into the $\mathrm{Si}$ spacer, resulting in Ge enrichment of the spacer layer and Ge depletion from the Ge layer. ${ }^{35-37}$ The expected Ge concentration gradually decreases from the Ge layer with a decay length of about 1-2 nm, depending on the deposited amount of Ge and the growth temperature. ${ }^{35-37}$ When the period length is shorter or comparable to the decay length of the Ge segregation, the Ge nanodot layers will be separated by SiGe layers rather than pure Si. The average Ge concentration of the spacer increases with further decreasing the period length, which may result in a decrease of acoustic impedance mismatch of the interfaces, thus leading to a weakening of the interface effect on the phonon transport and to a reduced $\rho$. Second, when the period length is comparable to the height of the nanodots, the nanodots may be physically in touch with each other in the growth direction. ${ }^{38}$ We speculate that the connection of nanodots may provide some SiGe channel for phonon transport without interface scattering, thus reducing the interface effect and $\rho$. In order to investigate whether the nanodots are connected together in our NDSLs, we etched the samples by $\mathrm{NHH}$ solution [1:1 vol. $\left(28 \% \mathrm{NH}_{4} \mathrm{OH}: 31 \%\right.$ $\left.\mathrm{H}_{2} \mathrm{O}_{2}\right)$, which selectively removes $\mathrm{Si}_{1-\mathrm{x}} \mathrm{Ge}_{\mathrm{x}}$ over pure $\mathrm{Si}^{38-40}$

Figures 4(e) and 4(f) are AFM images of etched NDSLs with period length of $2.3 \mathrm{~nm}$ and $1.5 \mathrm{~nm}$, respectively. After etching in $\mathrm{NHH}$ solution for $1 \mathrm{~h}$, we find that the nanodots are removed and holes appear on the surface. The hole density is lower than that of nanodot before the etching for the sample with period length of $2.3 \mathrm{~nm}$, while the densities are comparable for the sample with period length of $1.5 \mathrm{~nm}$. Figs. 4(g) and 4(h) show representative linescans of the images shown in Figs. 4(e) and 4(f) along the [110] direction. From the linescans, we find that the depth of the holes is less than $2 \mathrm{~nm}$ for the sample with period length of $2.3 \mathrm{~nm}$, while for the sample with period length of $1.5 \mathrm{~nm}$, the hole depth is mostly larger than $2 \mathrm{~nm}$. The real depth may be larger than the measured value because of the convolution effect of the AFM tip. This indicates that when the period length is $1.5 \mathrm{~nm}$, the nanodots are connected together in the growth direction. The comparable density of holes and nanodots suggests that the WLs are still separated by the spacers, which is confirmed by the TEM image in the inset of Fig. 4(d). However, the connection of nanodots in subsequent layers could weaken the interface effect on phonon scattering, thus reducing $R_{\text {barrier }}$ and $\rho$ of the resulting SLs. Nevertheless, we do not observe any increase in $\kappa$. This means that the reduction of SL thickness $t_{S L}$ is faster than decreasing the $R_{\text {barrier }}$, as $\kappa=\frac{t_{S L}}{N \cdot R_{\text {barrier }}}$. The fact that we can reach a regime with vertically connected $\mathrm{SiGe}$ nanodots preserving very low $\kappa$ values makes such structure particularly interesting for further investigation on electrical transport properties. Concerning the vertically connected nanodots, we expect that hole transport through SiGe might become feasible. This might improve the situation as compared to the nanodots separated by pure Si spacer layers: since the bound hole-like states in the nanodots are quite deep, the spacer layer would prevent tunneling of holes between the SiGe nanodots. Using the structures with vertically connected nanodots instead, a conductive channel for holes might be created, ${ }^{41}$ and thus the Ge/Si NDSLs can also be studied under p-type conditions for the electrical transport. Futher investigation on the electrical transport is beyond the scope of this paper.

\section{B. Effect of nanodot density on the thermal conductivity}

In this section, we investigate the effects of the nanodots and WLs on phonons transport through the NDSLs. The investigated SLs consist of 21 Ge layers, which are separated by a $2.7 \mathrm{~nm}$-thick $\mathrm{Si}$ spacer, as sketched in Fig. 1(b). Figs. 6(a)-6(e) show the surface morphology of as-grown SLs with nanodot density varying from 0 to $8.3 \times 10^{10} \mathrm{~cm}^{-2}$. In order to maintain the nanodot density constant from the bottommost layer to the topmost layer, the deposited amount of Ge, $t_{G e}$ was gradually decreased in the first 4 periods and then was kept constant for the last 17 periods, following the approach in Ref. 23. The details of the growth protocols can be found in Table I. The nanodot density $\left(N_{N D}\right)$ can be well controlled by varying $t_{G e}$. Fig. 6(f) shows that $N_{N D}$ increases with average $t_{G e}$ up to $\sim 5.2 \AA$. The slight decrease for larger Ge amounts is attributed to the occurrence of larger dots, which consume part of the deposited Ge from smaller dots due to their lower surface chemical potential.

Figures 7(a) and 7(b) show the total thermal resistance $R_{S L}$ and $\kappa$ of the SL layers as a function of $N_{N D}$, respectively. By increasing $N_{N D}$ from 0 to $8.3 \times 10^{10} \mathrm{~cm}^{-2}, R_{S L}$ increases from $2.6 \times 10^{-8} \mathrm{~m}^{2} \cdot \mathrm{K} / \mathrm{W}$ to $4.3 \times 10^{-8} \mathrm{~m}^{2} \cdot \mathrm{K} / \mathrm{W}$ (see Fig. 7 (a)) and $\kappa$ decreases from 2.3 to $1.5 \mathrm{~W} / \mathrm{m} \cdot \mathrm{K}$ (see Fig. 7(b)). The decrease in $\kappa$ with increasing nanodot density is in line with Lee's results. ${ }^{17}$

According to our recent results on planar $\mathrm{Ge} / \mathrm{Si} \mathrm{SLs},{ }^{37}$ $R_{\text {barrier }}$ of the WL is a linear function of the deposited amount of $\mathrm{Ge} \quad\left(R_{\text {barrier }}=\rho_{W L} \cdot t_{G e}\right.$, where the "thermal resistivity" $\rho_{W L}$ is constant, at least for Si-spacer thicknesses 


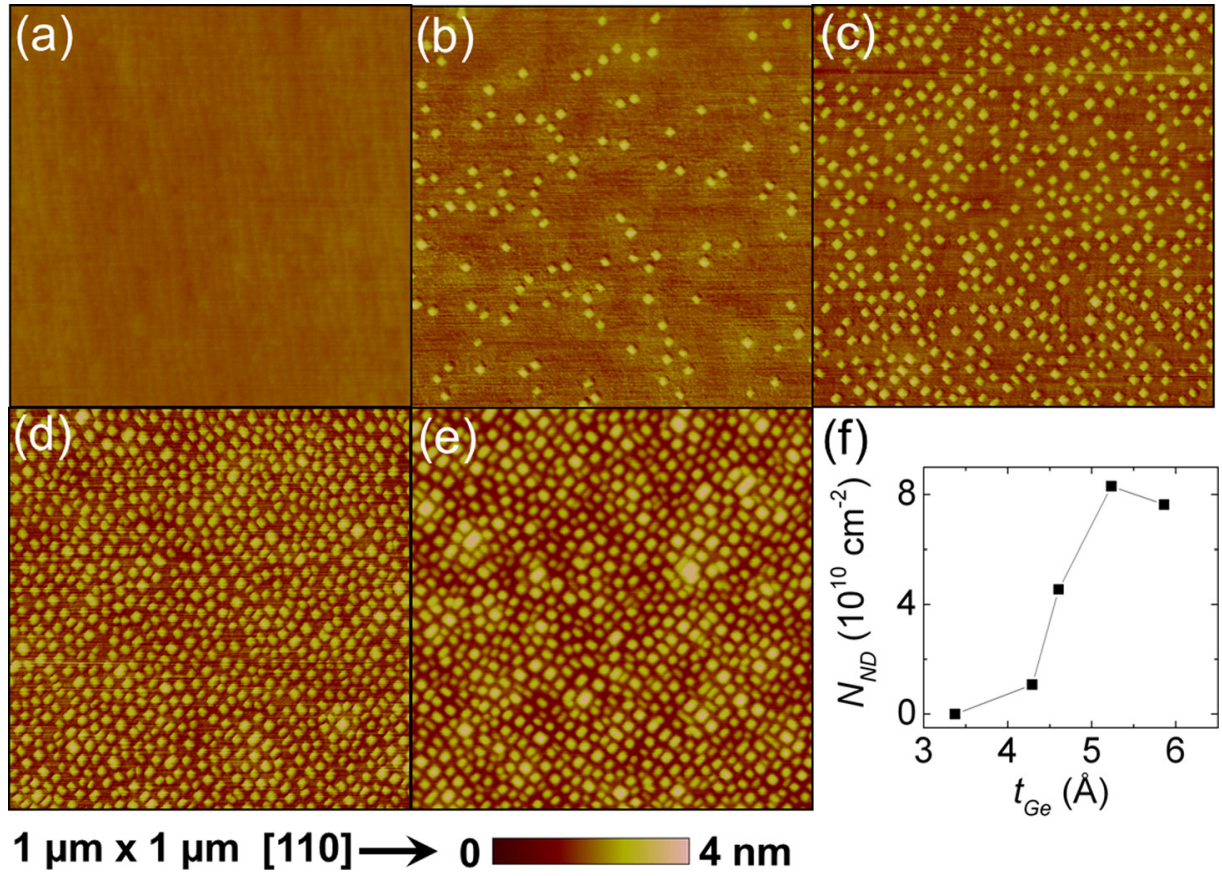

FIG. 6. (a-e) AFM images of asgrown superlattices with variable nanodot density and constant $\mathrm{Si}$ spacer thickness of $2.7 \mathrm{~nm}$ and 21 Ge layers. (f) Nanodot density as a function of the average deposited amount of $\mathrm{Ge}$ in the Ge layers, $t_{G e}$. larger than $\sim 1.5 \mathrm{~nm})$. In an attempt to discriminate the effects of WLs and nanodots, we define two quantities $\rho_{W L}$ and $\rho_{N D}$ to represent the contributions of Ge in the WLs and the nanodots, respectively to the barrier thermal resistance. We write the effective thermal resistivity as $\rho=\rho_{N D} \cdot \eta+\rho_{W L} \cdot(1-\eta)$, where $\eta$ is the fraction of the total deposited $\mathrm{Ge}$ in and under the nanodots. The values of $\rho_{\mathrm{WL}}$ and $\rho_{\mathrm{ND}}$ can be evaluated from the dependence of $\rho$ on $\eta$. (a)
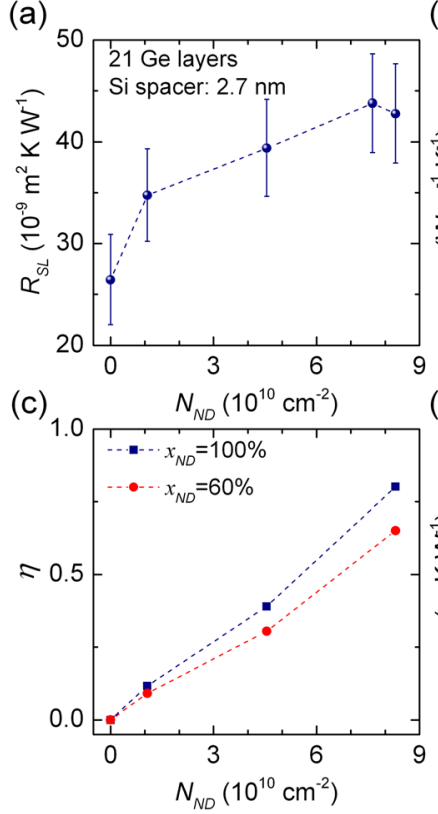

(b)

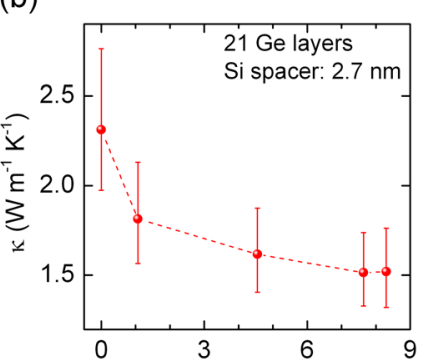

(d)

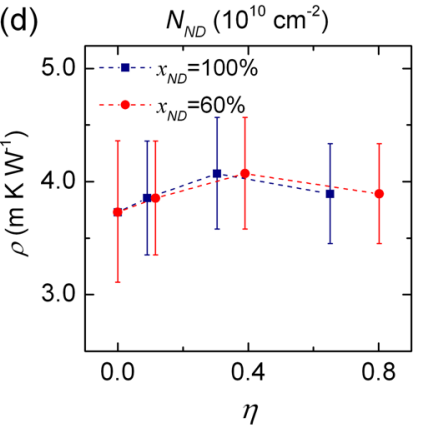

FIG. 7. Total thermal resistance $R_{S L}$ (a) and cross-plane thermal conductivity $\kappa$ (b) of Ge/Si SLs as a function of nanodot density $N_{N D}$. (c) Fraction $\eta$ of the total deposited amount of Ge in and under the nanodots as a function of $N_{N D}$. (d) Thermal resistivity $\rho$ of the SLs as a function of $\eta$. The blue squares and red circles in (c) and (d) correspond to NDs with estimated Ge concentration $x_{N D}$ of $100 \%$ and $60 \%$, respectively. The samples are $\mathrm{Ge} / \mathrm{Si}$ SLs with Si spacer thickness of $2.7 \mathrm{~nm}$ and 21 Ge layers (sample series 2).
We first estimate the value of $\eta$ as a function of the nanodot density. The nanodot density is supposed to be homogenous from the bottommost layer to the topmost layer. It is furthermore known that the nanodots have the shape of pyramids with square base and are bounded by four $\{105\}$ facets, ${ }^{23}$ so that their aspect ratio, i.e., height/base width is 0.1 . The height distribution of the nanodot can be obtained from AFM measurements on a sample surface with area $S=1 \mu \mathrm{m}$ $\times 1 \mu \mathrm{m}$. The total volume of the nanodots $V_{N D}$ equals the sum of the volumes of each nanodot, i.e., of $100 / 3 \cdot h^{3}$, where $h$ is the height of a single nanodot. (Note that $V_{N D}$ does not include the WL material located under the dots). In a single layer, the Ge amount (in unit of layer thickness) in the WL regions which is not covered by NDs can be estimated as

$$
t_{W L}=\left(1-S_{N D} / S\right)\left(t_{G e} \cdot S-V_{N D} \cdot x_{N D}\right) / S,
$$

where $S_{N D} / S$ is the fractional area occupied by the nanodots and $x_{N D}$ is the average Ge concentration in the nanodots. The Ge amount in and under the nanodots in a single period is $t_{N D}=t_{G e}-t_{W L}$, thus the fraction $\eta=t_{N D} / t_{G e}$.

Fig. 7(c) shows the dependence of $\eta$ on the nanodot density. With increasing the nanodot density, $\eta$ increases as a result of the increase in the fraction of the surface covered by nanodots and the incorporation of Ge from WL to nanodot during growth ${ }^{23,42}$ For the growth temperature of $500{ }^{\circ} \mathrm{C}$, the nanodots are expected to be alloyed to some extent. The effect of alloying on $\eta$ is illustrated in Fig. 7(c) by assuming average $\mathrm{Ge}$ concentrations, $x_{N D}$, as $100 \%$ or $60 \%$. Fig. 7(d) shows the calculated $\rho$ as a function of $\eta$. It is interesting to find that all $\rho$ 's are comparable within error bars and insensitive to variations of $\eta$. According to the equation $\rho=\rho_{N D} \cdot \eta+\rho_{W L} \cdot(1-\eta)=\left(\rho_{N D-} \rho_{W L}\right) \cdot \eta+\rho_{W L}$, we infer that $\rho_{N D}$ is similar to $\rho_{W L}$ and that their values are about $3.8 \mathrm{~m} \cdot \mathrm{K} / \mathrm{W}$. This result suggests that no matter the deposited $\mathrm{Ge}$ is in the WLs or in the nanodots, its contribution to the barrier thermal resistance is similar. This conclusion is rather 


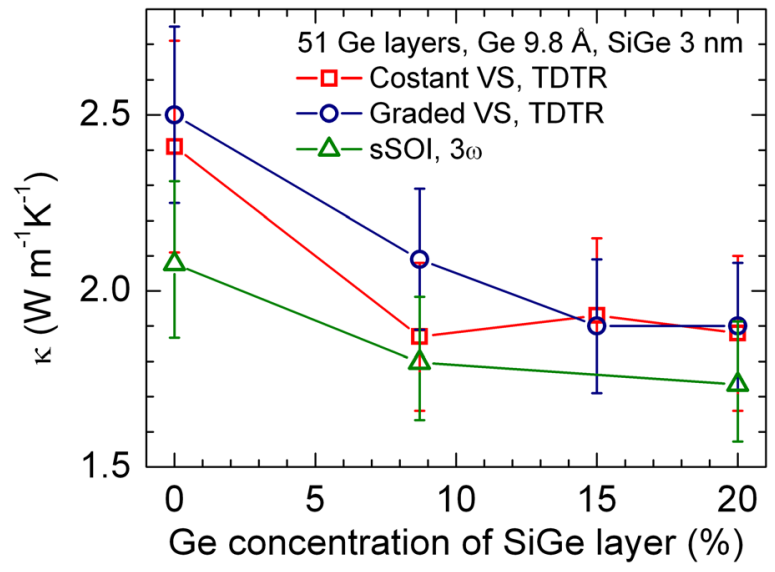

FIG. 8. Thermal conductivity $\kappa$ of Ge/SiGe NDSLs as a function of Ge concentration of the SiGe spacer layer. The Ge/SiGe NDSLs are grown on constant composition virtual substrates (VS) (red squares), graded VS (blue circles), and strained silicon-on-insulator (sSOI) substrates (green triangles), respectively. The superlattices grown on VS are measured by the timedomain thermoreflectance (TDTR) method, the superlattices grown on sSOI substrates are measured by the differential $3 \omega$ method.

surprising as one may expect nanodots to affect phonon scattering differently from a planar WL. A possible reason is that the already shallow nanodots considered here (with a height/base ration of $\sim 0.1$ ) may be further flattened during their overgrowth with Si. For instance, Ref. 43, reported a decrease of aspect ratio down to $\sim 0.03$ after capping of $\mathrm{Ge}$ nanodots with only $4 \mathrm{ML}$ of $\mathrm{Si}$ at $450{ }^{\circ} \mathrm{C}$. From this point of view, the difference between $\mathrm{WL}$ and nanodot materials is small.

\section{Effect of Ge concentration in the matrix on the thermal conductivity}

In this section, we investigate the effect of the Ge concentration in the spacer layers on the reduction of the thermal conductivity of Ge/SiGe NDSLs. The Ge/SiGe NDSLs were grown on different kinds of substrates, as schematically illustrated in Fig. 1(c). The deposited amount of Ge per layer is $9.8 \AA$, and the thickness of the SiGe spacer is kept constant and equal to $3 \mathrm{~nm}$. During growth, the only variable parameter is the Ge concentration of the SiGe spacer. The nanodot density of the SLs is between $0.8 \times 10^{10} \mathrm{~cm}^{-2}$ and $2.1 \times 10^{10} \mathrm{~cm}^{-2}$. With increasing Ge concentration, more Ge will segregate from the SiGe layer to the Ge nanodot layer, resulting in an increase in size of nanodots. Because of the thick SiGe buffer layer on the virtual substrates $(1 \sim 4 \mu \mathrm{m})$ and the resultant large background for the differential $3 \omega$ measurement, we used time-domain thermoreflectance (TDTR $)^{44,45}$ measurements to determine the thermal conductivity of the NDSLs grown on virtual substrates. As compared to the differential $3 \omega$ measurement, TDTR confines temperature fluctuations to the Ge/SiGe NDSL layer by using a high measurement frequency (10 MHz). Therefore, the influence of the thick SiGe buffer becomes negligible. The details of TDTR measurement can be found in Refs. 45 and 46. For the Ge/SiGe NDSLs grown on sSOI wafer, instead, we used the differential $3 \omega$ with the two-layer method. Fig. 8 shows the $\kappa$ of the Ge/SiGe NDSLs as a function of Ge concentration of the SiGe layer. Although the $\mathrm{Ge} / \mathrm{SiGe}$ NDSLs are grown on three different substrates, increasing the Ge concentration of the SiGe layer from $0 \%$ to $20 \%$ consistently leads to a reduction of $\kappa$ by $\sim 20 \%$. This result is in line with the prediction in Ref. 47, as increasing the Ge concentration of the SiGe layer enhances scattering effect on the short-wavelength phonons.

An interesting aspect emerges when comparing the data shown in Fig. 8 and those shown in Fig. 5 (b): the thermal conductivity of samples with pure Si spacer grown on virtual buffers and sSOI appears systematically higher than the thermal conductivity of the sample with similar Si spacer thickness and Ge amount grown on $\mathrm{Si}$ (001). A possible reason is that Ge segregation, which is effective in reducing thermal conductivity, ${ }^{37}$ is more pronounced when growth is performed on $\mathrm{Si}(001)$ because of the larger misfit strain, or that strain itself increases the barrier resistance by affecting the acoustic mismatch between the layers composing the SLs. Further investigations are required to draw conclusions on this issue.

\section{CONCLUSIONS}

In conclusion, we have explored the limits of the crossplane thermal conductivity in superlattices made of $\mathrm{Ge} / \mathrm{Si}(\mathrm{Ge})$ layers with nanodots. When the period length is shorter than $2 \mathrm{~nm}$, a drastic drop of thermal resistance is found, revealing a weakening of interface effect on phonon transport for short period lengths. By comparing the thermal conductivity of NDSLs with variable nanodot density and SL with only WLs, it appears that the effect of nanodots is comparable with that produced by planar WLs. Finally, by replacing the Si spacer material with $\mathrm{SiGe}$ of variable concentration, we observed a drop in thermal conductivity with increasing Ge concentration in the spacer. This decrease is however offset by a raise of the thermal conductivity of superlattices with pure Si spacers grown on virtual substrates as compared to conventional Si (001) substrates.

\section{ACKNOWLEDGMENTS}

We are grateful to N. Katcho and N. Mingo for fruitful discussions on thermal transport through SiGe superlattices, David G. Cahill for his support with TDTR measurements, D. J. Thurmer, M. Glaser and F. Schäffler for assistance with the MBE growth, D. Grimm, I. Mönch, A. Halilovic, U. Kainz, and S. Bräuer for assistance with sample processing for $3 \omega$ measurements. This work was supported by the SPP1386 (RA 1634/5-1), EU FP7 (NEAT, Grant No. 263440 and GREEN Silicon Grant No. 257750), and Regione Lombardia through the grant: Dote ricercatori. TDTR measurements performed at the U. Illinois were supported by the US Air Force Office of Scientific Research (AFOSR) Multidisciplinary University Research Initiative Grant No. FA9550-08-1-0407.

\footnotetext{
${ }^{1}$ M. S. Dresselhaus, G. Chen, M. Y. Tang, R. Yang, H. Lee, D. Wang, Z. Ren, J.-P. Fleurial, and P. Gogna, Adv. Mate. 19, 1043 (2007).

${ }^{2}$ C. B. Vining, Nature Mater. 8, 83 (2009).
} 
${ }^{3}$ D. G. Cahill, W. K. Ford, K. E. Goodson, G. D. Mahan, A. Majumdar, H. J. Maris, R. Merlin, and S. R. Phillpot, J. Appl. Phys. 93, 793 (2003).

${ }^{4}$ G. J. Snyder and E. S. Toberer, Nature Mater. 7, 105 (2008).

${ }^{5}$ L. D. Hicks and M. S. Dresselhaus, Phys. Rev. B 47, 12727 (1993).

${ }^{6}$ M. Zebarjadi, G. Joshi, G. Zhu, G. B. Yu, A. Minnich, Y. Lan, X. Wang, M. S. Dresselhaus, Z. Ren, and G. Chen, Nano Lett. 11, 2225 (2011).

${ }^{7}$ G. Fiedler and P. Kratzer, New J. Phys. 15, 125010 (2013).

${ }^{8}$ T. Koga, S. B. Cornin, M. S. Dresselhaus, J. L. Liu, and K. L. Wang, Appl. Phys. Lett. 77, 1490 (2000).

${ }^{9}$ B. Poudel, Q. Hao, Y. Ma, Y. Lan, A. Minnich, B. Yu, X. Yan, D. Wang, A. Muto, D. Vashaee, X. Chen, J. Liu, M. S. Dresselhaus, G. Chen, and Z. Ren, Science 320, 634 (2008).

${ }^{10}$ G. Joshi, H. Lee, Y. Lan, X. Wang, G. Zhu, D. Wang, R. W. Gould, D. C. Cuff, M. Y. Tang, M. S. Dresselhaus, G. Chen, and Z. Ren, Nano Lett. 8, 4670 (2008).

${ }^{11}$ K. Biswas, J. He, I. D. Blum, C.-I. Wu, T. P. Hogan, D. N. Seidman, V. P. Dravid, and M. G. Kanatzidis, Nature 489, 414 (2012).

${ }^{12}$ A. Samarelli, L. F. Llin, S. Cecchi, J. Frigerio, T. Etzelstorfer, E. Müller, Y. Zhang, J. R. Watling, D. Chrastina, G. Isella, J. Stangl, J. P. Hague, J. M. R. Weaver, P. Dobson, and D. J. Paul, J. Appl. Phys. 113, 233704 (2013).

${ }^{13}$ L. F. Llin, A. Samarelli, S. Cecchi, T. Etzelstorfer, E. M. Gubler, D. Chrastina, G. Isella, J. Stangl, J. M. R. Weaver, P. S. Dobson, and D. J. Paul, Appl. Phys. Lett. 103, 143507 (2013).

${ }^{14}$ S. M. Lee, D. G. Cahill, and R. Venkatasubramanian, Appl. Phys. Lett. 70, 2957 (1997).

${ }^{15}$ S. T. Huxtable, A. R. Abramson, C.-L. Tien, A. Majumdar, C. LaBounty, X. Fan, G. Zeng, J. E. Bowers, A. Shakouri, and E. T. Croke, Appl. Phys. Lett. 80, 1737 (2002).

${ }^{16}$ W. Kim, J. Zide, A. Gossard, D. Klenov, S. Stemmer, A. Shakouri, and A. Majumdar, Phys. Rev. Lett. 96, 045901 (2006).

${ }^{17}$ M. L. Lee and R. Venkatasubramanian, Appl. Phys. Lett. 92, 053112 (2008).

${ }^{18}$ G. Pernot, M. Stoffel, I. Savic, F. Pezzoli, P. Chen, G. Savelli, A. Jacquot, J. Schumann, U. Denker, I. Mönch, Ch. Deneke, O. G. Schmidt, J. M. Rampnoux, S. Wang, M. Plissonnier, A. Rastelli, S. Dilhaire, and N. Mingo, Nature Mater. 9, 491 (2010).

${ }^{19}$ J. Garg and G. Chen, Phys. Rev. B 87, 140302 (2013).

${ }^{20}$ O. G. Schmidt, C. Lange, and K. Eberl, Appl. Phys. Lett.75, 1905 (1999).

${ }^{21}$ G. Chen, B. Sanduijav, D. Matei, G. Springholz, D. Scopece, M. J. Beck, F. Montalenti, and L. Miglio, Phys. Rev. Lett. 108, 055503 (2012).

${ }^{22}$ J. J. Zhang, G. Katsaros, F. Montalenti, D. Scopece, R. O. Rezaev, C. Mickel, B. Rellinghaus, L. Miglio, S. De Franceschi, A. Rastelli, and O. G. Schmidt, Phys. Rev. Lett. 109, 085502 (2012).

${ }^{23}$ U. Denker, H. Sigg, and O. G. Schmidt, Mater. Sci. Eng. B 101, 89 (2003).
${ }^{24}$ C. Rosenblad, H. R. Deller, A. Dommann, T. Meyer, P. Schroeter, and H. von Känel, J. Vac. Sci. Technol. A 16, 2785 (1998).

${ }^{25}$ O. Moutanabbir and U. Gösele, Annu. Rev. Mater. Res. 40, 469 (2010).

${ }^{26}$ A. Tarun, N. Hayazawa, H. Ishitobi, S. Kawata, M. Reiche, and O. Moutanabbir, Nano Lett. 11, 4780 (2011).

${ }^{27}$ D. G. Cahill, Rev. Sci. Instrum. 61, 802 (1990).

${ }^{28}$ D. G. Cahill, M. Kativar, and J. R. Abelson, Phys. Rev. B 50, 6077 (1994).

${ }^{29}$ T. Borca-Tasciuc, A. R. Kumar, and G. Chen, Rev. Sci. Instrum. 72, 2139 (2001).

${ }^{30}$ Y. K. Koh, S. L. Singer, W. Kim, J. M. O. Zide, H. Lu, D. G. Cahill, A. Majumdar, and A. C. Gossard, J. Appl. Phys. 105, 054303 (2009).

${ }^{31}$ J. L. Liu, A. Khitun, K. L. Wang, T. Borca-Tasciuc, W. L. Liu, G. Chen, and D. P. Yu, J. Cryst. Growth 227-228, 1111 (2001).

${ }^{32}$ A. Jacquot, B. Lenoir, A. Dauscher, M. Stölzer, and J. Meusel, J. Appl. Phys. 91, 4733 (2002)

${ }^{33}$ S.-M. Lee and D. G. Cahill, J. Appl. Phys. 81, 2590 (1997).

${ }^{34}$ Z. Wang and N. Mingo, Appl. Phys. Lett. 97, 101903 (2010).

${ }^{35}$ D. J. Godbey and M. G. Ancona, J. Vac. Sci. Technol. A 15, 976 (1997).

${ }^{36}$ M. Brehm, M. Grydlik, H. Lichtenberger, T. Fromherz, N. Hrauda, W. Jantsch, F. Schäffler, and G. Bauer, Appl. Phys. Lett. 93, 121901 (2008).

${ }^{37}$ P. Chen, N. A. Katcho, J. P. Feser, W. Li, M. Glaser, O. G. Schmidt, D. G. Cahill, N. Mingo, and A. Rastelli, Phys. Rev. Lett. 111, 115901 (2013).

${ }^{38}$ J. J. Zhang, N. Hrauda, H. Groiss, A. Rastelli, J. Stangl, F. Schäffler, O. G. Schmidt, and G. Bauer G, Appl. Phys. Lett. 96, 193101 (2010).

${ }^{39}$ A. Rastelli, M. Stoffel, A. Malachias, T. Merdzhanova, G. Katsaros, K. Kern, T. H. Metzger, and O. G. Schmidt, Nano Lett. 8, 1404 (2008).

${ }^{40}$ M. Stoffel, A. Malachias, T. Merdzhanova1, F. Cavallo, G. Isella, D. Chrastina, H. von Känel, A. Rastelli, and O. G. Schmidt, Semicond. Sci.Technol. 23, 085021 (2008).

${ }^{41}$ D. Grützmacher, T. Fromherz, C. Dais, J. Stangl, E. Mueller, Y. Ekinci, H. H. Solak, H. Sigg, R. T. Lechner, E. Wintersberger, S. Bimer, V. Holy, and G. Bauer, Nano Lett. 7, 3150 (2007).

${ }^{42}$ M. Brehm, F. Montalenti, M. Grydlik, G. Vastola, H. Lichtenberger, N. Hrauda, M. J. Beck, T. Fromherz, F. Schäffler, L. Miglio, and G. Bauer, Phys. Rev. B 80, 205321 (2009).

${ }^{43}$ M. Kummer, B. Vögeli, and H. von Känel, Mater. Sci. Eng. B 69, 247 (2000).

${ }^{44}$ C. A. Paddock and G. L. Eesley, J. Appl. Phys. 60, 285 (1986).

${ }^{45}$ D. G. Cahill, Rev. Sci. Instrum. 75, 5119 (2004).

${ }^{46}$ J. P. Feser, J. S. Sadhu, B. P. Azeredo, K. H. Hsu, J. Ma, J. Kim, M. Seong, N. X. Fang, X. Li, P. M. Ferreira, S. Sinha, and D. G. Cahill, J. Appl. Phys. 112, 114306 (2012).

${ }^{47}$ N. Mingo, D. Hauser, N. P. Kobayashi, M. Plissonnier, and A. Shakouri, Nano Lett. 9, 711 (2009). 